\title{
Pricing-Enabled QoS for UMTS/WLAN Network
}

\author{
Jang, Hung-Chin Lu, Bohan \\ Department of Computer Science, National ChengChi University, Taiwan, R.O.C.
}

\begin{abstract}
In this paper we propose a pricing-enabled QoS for the hybrid UMTS/WLAN network. The proposed methodology is an integration of usage-based, non usage-based, static, and dynamic pricing schemes and DiffServ (Differentiated Services). We propose the utility function and cost function for our needs, and the pricing mechanism is based on differentiated service classes. By the affection of price, a user could express the service class he actually needs and expects. Network resources could be allocated appropriately to each service class avoiding inappropriate allocation and wastage, and thus meet the QoS requirement.
\end{abstract}

Keywords : Pricing, Quality of Service (QoS), DiffServ

\section{Introduction}

In recent years, economic pricing theory has been applied to communication service market. With this, service providers could provide enough incentives for users to use network resources more efficiently and thus realize the potential preferences of users. In this research, we study how to apply the economics to enhance the management and allocation of network resources to meet the QoS requirement. Besides, we improve providers' revenue by using economic analysis. We believe that the increasing revenue would give providers incentive to invest more in development of network infrastructure and related researches. This virtuous circle will definitely contribute to the development of whole communication network and derivative services.

To simplify the real problem, many proposed researches usually assumed that the market is perfect competition market, and there is no consideration of the integration of heterogeneous networks. Besides, most of the researches are verified by the mathematical analysis without simulation experiments. However, if we look at the telecommunication markets, the majority are oligopoly markets, and the existing providers do have influences on the markets. On the other hand, the integration of heterogeneous networks is the trend of communication market in the near future. In this research, we will propose the utility function and cost function, and use the third-degree price discrimination and two-part tariff to enhance the QoS for UMTS/WLAN integrated network. Finally, we will verify the proposed method by simulation and evaluate the performance in terms of delay of each service class. The rest of the paper is organized as follows. Section 2 proposes our pricing strategies and QoS service class mapping, utility function, cost function, third-degree price discrimination and two-part tariff. Section 3 shows some simulation results. Section 4 concludes this research.

\section{Pricing Strategy and Service Class Mapping}

In this section, we will justify how the proposed pricing strategy would enhance the QoS. First, we introduce how the DiffServ would operate in UMTS/WLAN integrated network. Second, we define the mapping between QoS classes in UMTS/WLAN and DiffServ PHBs. Third, we propose the utility function of the consumer and cost function of the manufacture in detail. Finally, we elaborate on how the utility function, cost function, and QoS service class mapping would work with third-degree price discrimination and two-part tariff.

\subsection{UMTS/WLAN with DiffServ}

In this research, we adopt the tight coupling scheme as our integrated UMTS/WLAN network structure. We would describe how UMTS/WLAN would interwork with DiffServ in the following. Before we go on, we assume that the UE (User Equipment) and GGSN (Gateway GPRS Support Node) would support the edge functions of DiffServ, and the backbone IP network is also DiffServ enabled.

The overall end-to-end resource management structure for QoS could be divided into three layers. The highest and lowest layers are the IP and RAB (Radio Access Bearer) manager, respectively. The middle layer is the UMTS BS (Base Station) manager. DiffServ mechanism would be combined within IP manager layer and this manager is unaware of the underlying transport technologies. The mapping of QoS requirement between DiffServ and UMTS QoS management mechanism should also be defined in the UMTS BS manager layer. UMTS QoS control mechanism supports per-flow resource allocation and other control. This would be achieved by employing UMTS PDP context. Beyond the UMTS GGSN in the external IP network, it is expected that the evolving IETF standards DiffServ would provide sufficient QoS control and traffic engineering for various services.

The end-to-end QoS is provided by a local mechanism in the UE, the PDP Context over the UMTS access network, DiffServ through the backbone IP network, and DiffServ in the remote access network. Fig. 1 shows the operation of DiffServ 
in UMTS, the same concept is applicable to UMTS/WLAN network.

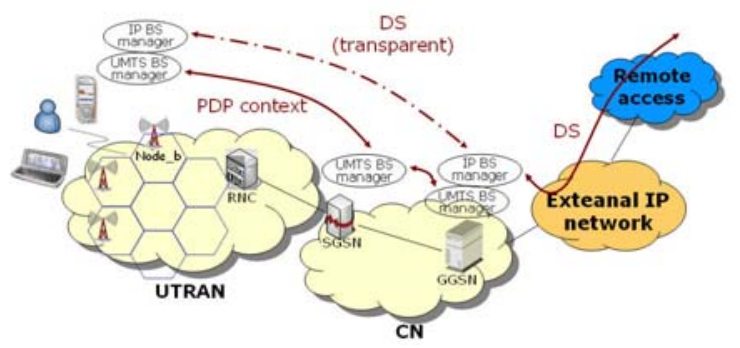

Fig. 1 : End-to-end all-IP scenario with DiffServ [3]

\subsection{QoS Class Mapping}

The QoS classes mapping between UMTS, WLAN and DiffServ, respectively, are required to be adapted to the DiffServ structure.

\begin{tabular}{|c|c|c|c|c|c|c|c|}
\hline \multicolumn{2}{|c|}{ PHB } & \multicolumn{6}{|c|}{ DSCP } \\
\hline \multicolumn{2}{|l|}{ EF PHB } & \multicolumn{6}{|c|}{101110} \\
\hline \multicolumn{2}{|l|}{ AF PHB } & \multicolumn{2}{|c|}{ Class 1} & \multicolumn{2}{|c|}{ Class 2} & Class 3 & Class 4 \\
\hline \multicolumn{2}{|c|}{ Low drop precedence } & AF11 & (001 010) & $A F 21$ & $(010010)$ & AF31 (011 010) & AF41 $\left(\begin{array}{lll}100 & 010\end{array}\right)$ \\
\hline \multicolumn{2}{|c|}{ Medium drop precedence } & AF12 & (001 100) & AF22 & $\left(\begin{array}{ll}010 & 100\end{array}\right)$ & AF32 (011 100) & AF42 $\left(\begin{array}{lll}100 & 100\end{array}\right)$ \\
\hline \multicolumn{2}{|c|}{ High drop precedence } & AF13 & (001 110) & AF23 & $\left(\begin{array}{lll}010 & 110\end{array}\right)$ & AF33 (011 110) & AF43 $(100 \quad 110)$ \\
\hline \multicolumn{2}{|l|}{ Best effort } & \multicolumn{6}{|c|}{000000} \\
\hline & \multicolumn{2}{|c|}{ Traffic class } & \multicolumn{2}{|c|}{ Delay bound } & & Traffic class & Delay bound \\
\hline \multirow[t]{4}{*}{ UMTS } & \multirow{2}{*}{\multicolumn{2}{|c|}{$\begin{array}{l}\text { Conversational } \\
\text { Interactive }\end{array}$}} & \multirow{2}{*}{\multicolumn{2}{|c|}{$\begin{array}{c}400 \mathrm{~ms} \\
4 \mathrm{~s}\end{array}$}} & \multirow[t]{2}{*}{ WLAN } & Voice & \multirow{4}{*}{$\begin{array}{c}400 \mathrm{~ms} \\
4 \mathrm{~s}\end{array}$} \\
\hline & & & & & & Video & \\
\hline & \multicolumn{2}{|c|}{ Streaming } & \multicolumn{2}{|c|}{$10 \mathrm{~s}$} & & Best effort & \\
\hline & \multicolumn{2}{|c|}{ Background } & & & & Background & \\
\hline
\end{tabular}

Table 1 : Proposed QoS Class Mapping

The QoS class mapping among UMTS, WLAN and DiffServ are summarized in Table 1 . We classify the Conversational class of UMTS and the Voice class of WLAN into EF PHB. Besides, the Interactive class of UMTS and the Video class of WLAN are classified into AF42 PHB. The Streaming class of UMTS and the Best-effort class of WLAN are classified into AF33 PHB. This mapping is referred to the method used by RADIUS. The lowest Background classes are classified into Best-effort PHB.

\subsection{Utility Function}

The utility function consists of the common and idiosyncratic tastes. Common taste is a function expressed by $\mathrm{v}(\cdot)$ ). It denotes the observable utility that any user could obtain from the consumption of the same product. Idiosyncratic taste, denoted by a variable $\varepsilon$, is the unobservable utility that a user would have from the consumption of the same product. We also add a positive parameter $\mu$ for $\varepsilon$ to show the weight of idiosyncratic taste over the total taste. The larger is the $\mu$, the more important is the idiosyncratic taste. The overall utility of each user could be expressed by equation (1).

$$
\mathrm{u}(\cdot)=\mathrm{v}(\cdot)+\mu \cdot \varepsilon_{\mathrm{i}}
$$

Here we define the common taste function as follows,

$\mathrm{v}(\cdot)=\mathrm{a}_{\mathrm{i}}+\left(\mathrm{I}_{\mathrm{i}} \cdot \mathrm{cs} \cdot \mathrm{rb}\right) \cdot\left(\ln \left(\frac{\mathrm{x}_{\mathrm{i}}}{\mathrm{rb} \cdot \mathrm{rt}}\right)\right)^{2}-\mathrm{p}_{\mathrm{i}}^{\mathrm{T}}-\left(\mathrm{S}_{\mathrm{i}} \cdot \mathrm{cs} \cdot \mathrm{ad}_{\mathrm{i}} \cdot \mathrm{rb}\right) \cdot \ln \left(\frac{\mathrm{x}_{\mathrm{i}}}{\mathrm{rb} \cdot \mathrm{rt}}\right)(2)$

$\mathrm{i}:$ the service class

$\mathrm{x}$ : the bandwidth that a user consumes or obtains (kbps)

$\mathrm{a}_{\mathrm{i}}$ : the basic utility that users will obtain in each service class

$I_{i}$ : the index of the service class, if $i>j$ then $I_{i}>I_{j}$

cs : cell scale factor, here we assume that umts $=1$, wlan $=0.1$

rb : the factor of bandwidth requirement of selected application

$\mathrm{rt}$ : the buffered factor

$\mathrm{p}^{\mathrm{T}}{ }_{\mathrm{i}}$ : the fixed charge of each class

$\mathrm{S}_{\mathrm{i}}$ : used to parameterize the sensitivity of each class to either congestion or delay

$\mathrm{ad}_{\mathrm{i}}:$ average delay of each class

$\left(S_{i} \cdot c s \cdot a d_{i} \cdot r b\right) \cdot \ln \left(\frac{x_{i}}{r b \cdot r t}\right):$ the congestion cost of each user.

In order to calculate the total demand functions of each service class, we further assume the number of users of each service class in the cells of UMTS and WLAN as follows.

\begin{tabular}{|l|c|c|c|c|}
\hline \multicolumn{5}{|c|}{ The forecasted number of users } \\
\hline & \multicolumn{2}{|c|}{ UMTS (20) } & \multicolumn{2}{c|}{ WLAN (20) } \\
\hline EF & $50 \%$ & 10 & $20 \%$ & 4 \\
\hline AF42 & $20 \%$ & 4 & $35 \%$ & 7 \\
\hline AF33 & $20 \%$ & 4 & $35 \%$ & 7 \\
\hline BE & $10 \%$ & 2 & $10 \%$ & 2 \\
\hline
\end{tabular}
cell of UMTS and WLAN

We assume that half the users are using voice application in UMTS and more than half the users are using video phone and multimedia streaming applications in WLAN. The total number of users in each cell is set to 20 . According to the above assumptions, the demand function of each service class can be calculated according to equation (3), where the parameter $n_{i}$ is the number of users of service class $i$.

Total_D $\left(\mathrm{x}_{\mathrm{i}}\right)=2 \cdot\left(\mathrm{I}_{\mathrm{i}} \cdot \mathrm{cs} \cdot \mathrm{rb} \cdot \mathrm{n}_{\mathrm{i}}\right) \cdot \frac{\ln \left(\frac{\mathrm{x}_{\mathrm{i}}}{\mathrm{rb} \cdot \mathrm{rt} \cdot \mathrm{n}_{\mathrm{i}}}\right)}{\mathrm{x}_{\mathrm{i}}}-\left(\mathrm{S}_{\mathrm{i}} \cdot \mathrm{cs} \cdot \mathrm{ad}_{\mathrm{i}} \cdot \mathrm{rb} \cdot \mathrm{n}_{\mathrm{i}}\right) \frac{1}{\mathrm{X}_{\mathrm{i}}}$

\subsection{Cost Function}

We define the cost function by using the envelope theorem as follows:

$$
\mathrm{TC}=\operatorname{Cost}(\mathrm{x}, \mathrm{k})=\mathrm{x} \cdot \mathrm{f}\left(\frac{\mathrm{x}}{\mathrm{k}}\right)+\mathrm{g}(\mathrm{k})
$$

$\mathrm{x}$ : bandwidth (kbps)

$\mathrm{k}$ : the capacity of the link (kbps)

$\mathrm{FC}=\mathrm{g}(\mathrm{k}):$ Fixed Cost, means the rent of link

$\mathrm{TVC}=\mathrm{x} \cdot \mathrm{f}\left(\frac{\mathrm{x}}{\mathrm{k}}\right)$ : Total Variable Cost, means the total

$$
\text { congestion cost }
$$

$\mathrm{AVC}=\mathrm{f}\left(\frac{\mathrm{x}}{\mathrm{k}}\right)$ : Average Variable Cost

$\mathrm{MC}=\frac{\Delta \mathrm{TVC}}{\Delta \mathrm{x}}=\frac{\partial \mathrm{TC}}{\partial \mathrm{x}}:$ Marginal Cost 
The total cost function consists of parameters $\mathrm{x}$ and $\mathrm{k}$, which are the bandwidth and capacity of the link respectively. We then define the marginal cost function as equation (5), where BSCR stands for Base Station Cost Ratio.

$$
\mathrm{MC}=\frac{\mathrm{BSCR}}{2000 \cdot \mathrm{k} \cdot\left(1-\frac{\mathrm{x}}{\mathrm{k}}\right)^{2}}
$$

\subsection{Third-Degree Price Discrimination and Two-Part Tariff}

The approximation of two-part tariff is based on Fig. 2 and formulae (6) to (8).

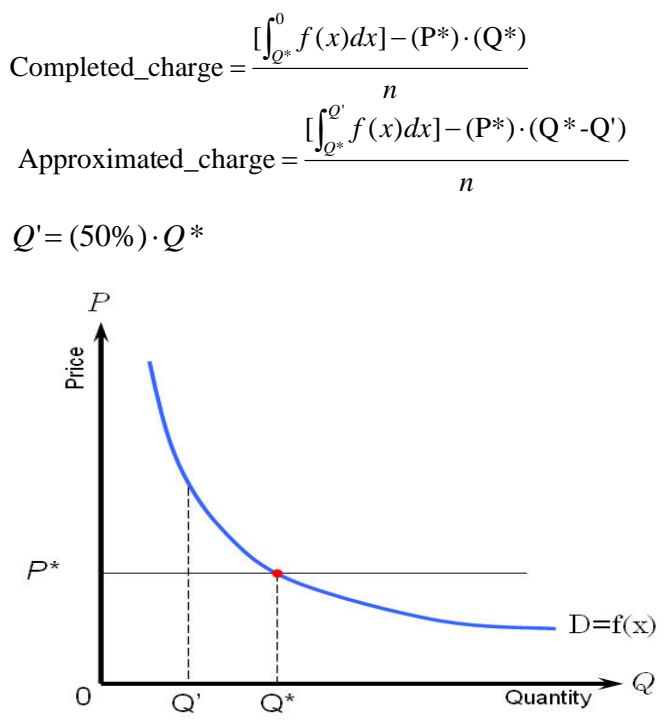

Fig. 2 : Approximation of two-part tariff

The final results of allocation bandwidth, discrimination price and fixed charge of two-part tariff are shown in Tables 3 and 4 .

\begin{tabular}{|l|c|c|c|c|}
\hline \multicolumn{2}{|c|}{ Discrimination price and allocation bandwidth calculation } \\
\hline & \multicolumn{2}{|c|}{ UMTS } & \multicolumn{2}{c|}{ WLAN } \\
\hline & $\mathrm{x}$ & $\mathrm{P}(\$ / \mathrm{kb})$ & $\mathrm{x}$ & $\mathrm{P}(\$ / \mathrm{kb})$ \\
\hline EF & 399 & 0.00281 & 139 & 0.00031 \\
\hline AF42 & 285 & 0.00275 & 433 & 0.00030 \\
\hline AF33 & 215 & 0.00242 & 327 & 0.000027 \\
\hline BE & 1 & 0.00082 & 1 & 0.00009 \\
\hline
\end{tabular}

Table 3 : Allocation bandwidth and discrimination price

\begin{tabular}{|l|c|c|}
\hline \multicolumn{3}{|c|}{ Two part tariff price calculation } \\
\hline & UMTS & WLAN \\
\hline EF & 0.501 & 0.105 \\
\hline AF42 & 0.578 & 0.134 \\
\hline AF33 & 0.508 & 0.094 \\
\hline BE & 0 & 0 \\
\hline
\end{tabular}

Table 4 : Fixed charge of two-part tariff

\section{Simulation and Analysis}

To evaluate the effect of bandwidth allocation based on our pricing mechanism, we perform two experimental scenarios. The only difference is the bandwidth allocation of each service class. In scenario 1 , the bandwidth allocation of each service class is determined by our pricing mechanism. Those of scenario 2 is determined by the user ratio. Table 5 shows the allocated bandwidth of each service class in these two scenarios.

\begin{tabular}{|l|c|c|c|c|}
\hline \multicolumn{5}{|c|}{ Allocation bandwidth } \\
\hline & \multicolumn{2}{|c|}{ Scenario 1 } & \multicolumn{2}{c|}{ Scenario 2 } \\
\hline & UMTS & WLAN & UMTS & WLAN \\
\hline EF & 399 & 139 & 450 & 180 \\
\hline AF42 & 285 & 433 & 180 & 315 \\
\hline AF33 & 215 & 327 & 180 & 315 \\
\hline BE & 1 & 1 & 90 & 90 \\
\hline
\end{tabular}

Table 5 : Allocation bandwidth of each service class in two scenarios

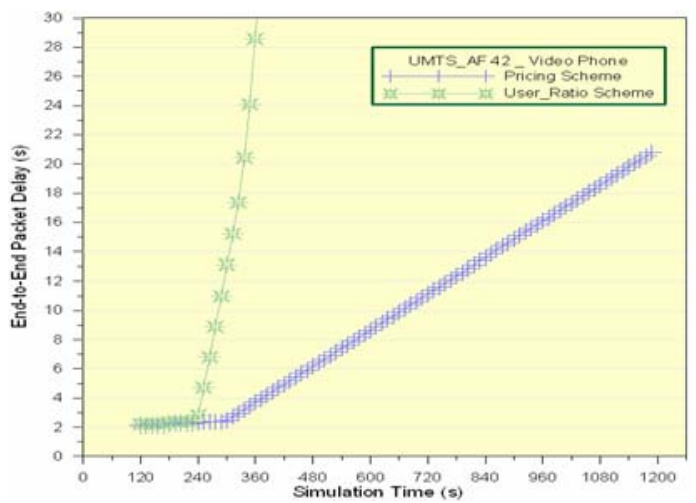

Fig. 3 : E2E packet delay of UMTS video phone application of two scenarios

Fig. 3 shows the packet end-to-end delay of UMTS video phone application of two scenarios. Because in both scenarios the allocated bandwidth is insufficient to support the highest traffic load when all users have performed the application, delay time would not fulfill the QoS requirement when traffic load exceeds the allocated bandwidth. In both scenarios, delay time keeps stable in about 2s and then rapidly increases when the traffic load exceeds total allocated bandwidth, which are after simulation time 300 s and 240s, respectively. Both scenarios did not allocate enough bandwidth so delay time would finally exceed 4s. Our method (scenario1) could efficiently slow down the rate of increase in delay time about $80 \%$ and extend the possible service time with QoS for 50\% comparing to that of scenario 2 in the worst-case. 


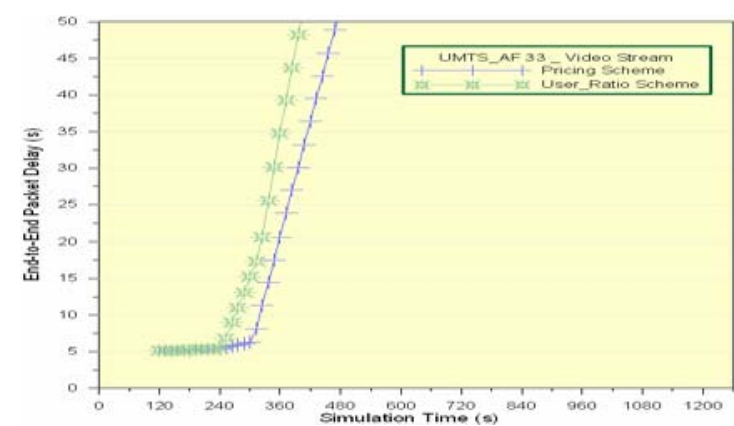

Fig. 4 : E2E packet delay of UMTS video stream application of two scenarios

Fig. 4 shows the packet end-to-end delay of UMTS video stream application of two scenarios. It is similar to the previous AF42 class. Both scenarios did not allocate enough bandwidth. The allocated bandwidth is insufficient to support the highest traffic load when total users have performed the application. In both scenarios, delay time keeps stable in about $6 \mathrm{~s}$ and then rapidly increases when the traffic load exceeds the allocated bandwidth, which are after simulation time 300s and 240s, respectively. Delay time would not fulfill the QoS requirement and finally exceeds 10 s when traffic load exceeds the allocated bandwidth. Our method (scenario1) could still slow down the rate of increase in delay time about $30 \%$ and extend the possible service time with QoS for 25\% comparing to that of scenario 2 in the worst-case.

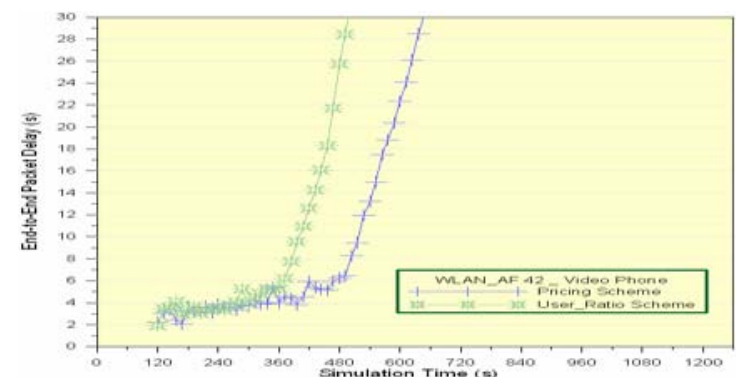

Fig. 5 : E2E packet delay of WLAN video phone application of two scenarios

Fig. 5 shows the packet end-to-end delay of WLAN video phone application of two scenarios. Although our method (scenario1) is able to allocate more bandwidth to WLAN AF42 class than scenario 2 does, the bandwidth is still insufficient to support the highest traffic load when all users have performed the application. The delay time would not fulfill the QoS requirement when traffic load exceeds the allocated bandwidth. Delay time is less than 4 s and increases slowly before $300 \mathrm{~s}$ in both scenarios. Delay time of scenario 2 begins to increase rapidly after 300s because the bandwidth is now unsatisfactory. In scenario 1 , this situation would take place until 420s. We could see that our method has less delay time at any simulation time comparing to that of scenario 2. Furthermore, it also slows down the rate of increase in delay time about $60 \%$ and extends the possible service time with QoS for $40 \%$ comparing to that of scenario 2 in the worst-case.

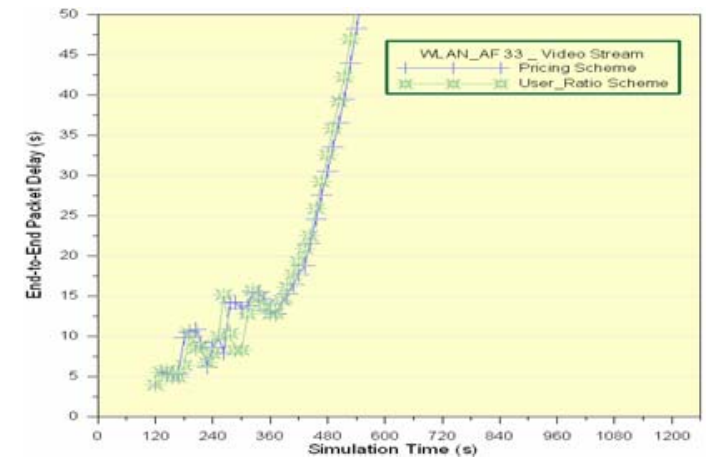

Fig. 6 : E2E packet delay of WLAN video stream application of two scenarios

Fig. 6 shows the packet end-to-end delay of WLAN video stream application of two scenarios. Both scenarios do not have enough bandwidth. The allocated bandwidth is insufficient to support the highest traffic load when total users have performed the application. The delay time would not fulfill the QoS requirement and finally exceeds 10 s when traffic load exceeds the allocated bandwidth. Simulation results of two scenarios in this class are very close. Delay time exceeds the bound of $10 \mathrm{~s}$ at simulation time about 300s and 240s in scenario 1 and 2, respectively. However, our method (scenario1) still has some improvements. It slows down the rate of increase in delay time about $10 \%$ and extends the possible service time with QoS for $25 \%$ comparingto that of scenario 2 in the worst-case.

\section{Conclusions}

This research applied economic pricing strategy with DiffServ to integrated UMTS/WLAN network to meet the QoS requirement. With limited bandwidth, the system is able to allocate suitable network resources to each service class using pricing theory. The simulations showed that the system can not only make users use network resources more efficiently but also increase the revenue of manufactures. This virtuous circle will certainly contribute to the development of whole communication network and derivative services.

\section{References}

[1] Christoph Lindemann, Marco Lohmann, and Axel Thümmler, "A unified approach for improving QoS and provider revenue in 3G mobile network," Mobile Networks and Applications 8, pp. 209-21, 2003.

[2] Costas Couroubetis and Richard Weber, Pricing Communication Networks Economics, Technology and Modelling, Wiley Press, 2003.

[3] Sudhir Dixit, and Ramjee Prasad, Wireless IP and Building the Mobile Internet, Artech House Press, 2002. 